\title{
The future of 3D printing of human tissues is taking shape
}

f.the ITOP
system
overcomes
previous
limitations
in tissue
size, shape,
structural
integrity, cell
survival and
vascularization
of bioprinted
tissue
constructs

The demand for artificial tissues is high owing to a shortage of donor organs, but developing such tissues for clinical applications is challenging. Anthony Atala and colleagues have now made substantial progress in 3D printing of tissues of clinically relevant size and structural integrity by interlacing microchannels, thus improving survival of the embedded cells.

The custom designed Integrated Tissue and Organ Printing (ITOP) system consists of a sophisticated nozzle system with a resolution of 2-50 $\mu \mathrm{m}$ that deposits cell-laden hydrogels together with biodegradable polymers. The cell matrix - consisting of gelatin, fibrinogen, hyaluronic acid and glycerol - is optimized to promote cell survival and differentiation whilst providing support and uniform dispersion. Mechanical shape and strength is provided by poly( $\varepsilon$-caprolactone) polymers, which were chosen for their low melting temperature,

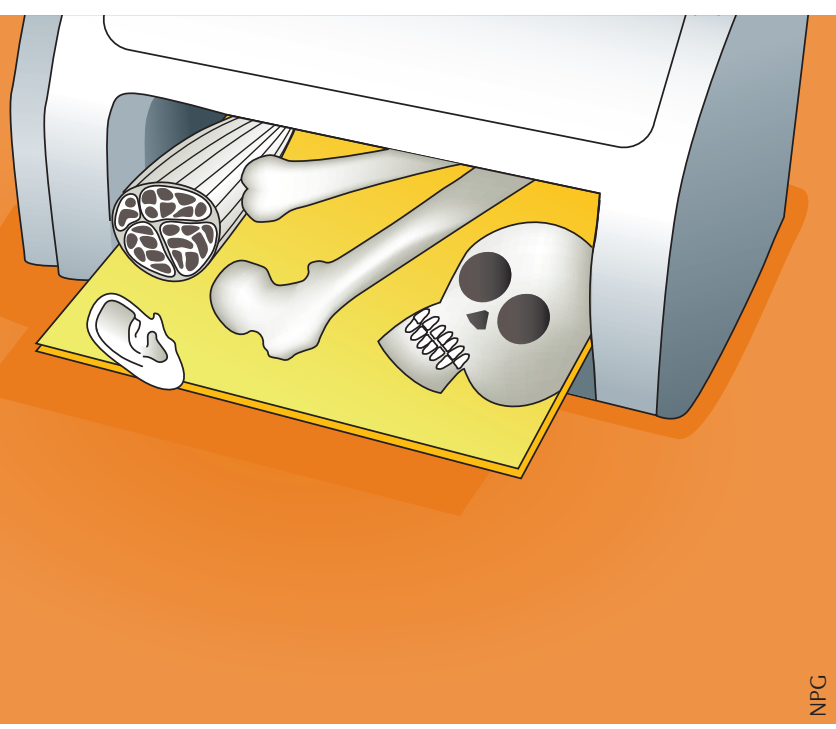

and an external Pluronic F-127 layer. After printing, the addition of a thrombin solution induces crosslinking of the fibrinogen and the unlinked components, including the bio-incompatible Pluronic F-127 layer, are then washed away. Different printing patterns based on the placement of the polymer were developed and were aimed at achieving either a stable structure or protection against external load. To accurately shape the tissue constructs, CT or MRI data were used as input to drive a motion-generating software program.

"The most significant finding is the ability to maintain cell viability in a large tissue construct, possibly larger than any that have been produced by 3D printing technology thus far," explains Daniel Grande, who is not an author on this study. Indeed, the maximum diffusion distance of nutrients and oxygen is just $100-200 \mu \mathrm{m}$, which considerably limits the size of tissue constructs and thus their clinical applicability. Atala explains that the ITOP system overcomes these challenges by printing microchannels that enable nutrients and oxygen to diffuse into the artificial tissues and keep the cells alive whilst the tissues develop their own system of blood vessels.

As a proof of principle, the authors generated mandible and calvarial bone structures, ear cartilage and skeletal muscle. All tissues showed blood vessel infiltration and tissue formation at the centre of the constructs both in vitro and in vivo - much more than acellular scaffolds. Clinical-sized mandible constructs loaded with human amniotic fluid-derived stem cells showed $>90 \%$ cell viability 1 day after printing and calcium deposition after 28-day culture in osteogenic conditions. Orthotopic implantation of similar calvarial bone constructs in rats showed better vascularization and bone tissue formation after 5 months, compared with the scaffold alone. To test complex shapes, a human-shaped ear was reconstructed based on CT scans. The implanted rabbit ear cartilage cells showed appropriate differentiation and cartilage deposition after 5-week chondrogenic culture. Ectopic transplantation of the $3 \mathrm{D}$-printed ear in athymic mice for 2 months revealed maintenance of ear shape, cartilage deposition, cell differentiation and improvement of material strength, which suggest in vivo maturation of the construct.

Thus, the ITOP system overcomes previous limitations in tissue size, shape, structural integrity, cell survival and vascularization of bioprinted tissue constructs. "Although it is certainly a step in the right direction, the lack of material characterization might hamper clinical translation," warns Grande. At this point, it is not yet clear whether the material is safe enough to be implanted in humans. To overcome these issues, the research group led by Atala is currently focusing on the production of tissues for human application, in addition to optimizing the technique to build more complex tissues and solid organs involving several cell types.

Liesbet Lieben, Associate Editor Nature Reviews Disease Primers

ORIGINAL ARTICLE Kang, H.-W. et al. A 3D bioprinting system to produce human-scale tissue constructs with structural integrity. Nat. Biotech. http://dx.doi.org/10.1038/nbt.3413 (2016) 


\section{RESEARCH HIGHLIGHTS}

\section{CORRECTION}

The future of 3D printing of human tissues is taking shape

Nat. Rev. Rheumatol. doi: 10.1038/nrrheum.2016.29 (2016)

In the version of this Research Highlight originally published online, the DOI of the original paper by Kang et al. was incorrect. This error has been corrected for the print, HTML and PDF versions of the article. 